\title{
Yield of trans nasal flexible oesophagoscopy (TNO) biopsies of suspected upper aero-digestive tract (UADT) lesions
}

Mr Hassan Mohammed FRCS (ORL-HNS), Mr Marcos Del Pero FRCS (ORL-HNS), Mr Matthew Coates MRCS ENT, Mr Liam Masterson FRCS (ORL-HNS), Mr Ramez Nassif FRCS (ORL-HNS)

\section{Introduction:}

TNO is a relatively recent addition to the field of head and neck cancer. It has good safety record and does not require sedation. Availability of a working channel allows for biopsies of suspicious lesions

\section{Materials and methods:}

All patients in whom TNO-guided biopsies were attempted over 2 years period in ENT outpatient were included. Data recorded included patient demographic, site of the lesions, final histological diagnosis, need for further biopsy before treatment.

\section{Discussion:}

Outpatient awake procedures are attractive both in terms of cost and in terms of avoidance of general anesthesia. When compared with rigid endoscopy, our results suggest favourable complication rate and comparable abandonment rate.

When comparison is made with OGD the success rate seems to be slightly better.

It is also noteworthy that TNO does not require general anaesthesia or sedation in contrast to rigid endoscopy and OGD respectively.

\section{Results:}

The study included 134 procedures on 121 patients. Of these, there were 19 abandoned procedures. This leaves 115 successful procedure where biopsies were taken, giving an overall procedure success (of obtaining biopsies) rate of $86 \%$. All further analysis will be made on these 115 procedures.

In 13 patients additional procedure was necessary. Four of them because of associated neck lump ( 3 FNA/ 1 core), 6 because of high clinical suspicion ( 3 tongue base) and 4 because TNO biopsy was not diagnostic. 4 out of 9 who were rebiopsied from primary site had SCC.

TNO-guided biopsy sites and subsites

Glottis

Tongue base

$\square$ PNS

Supraglottic

- Oesophageus

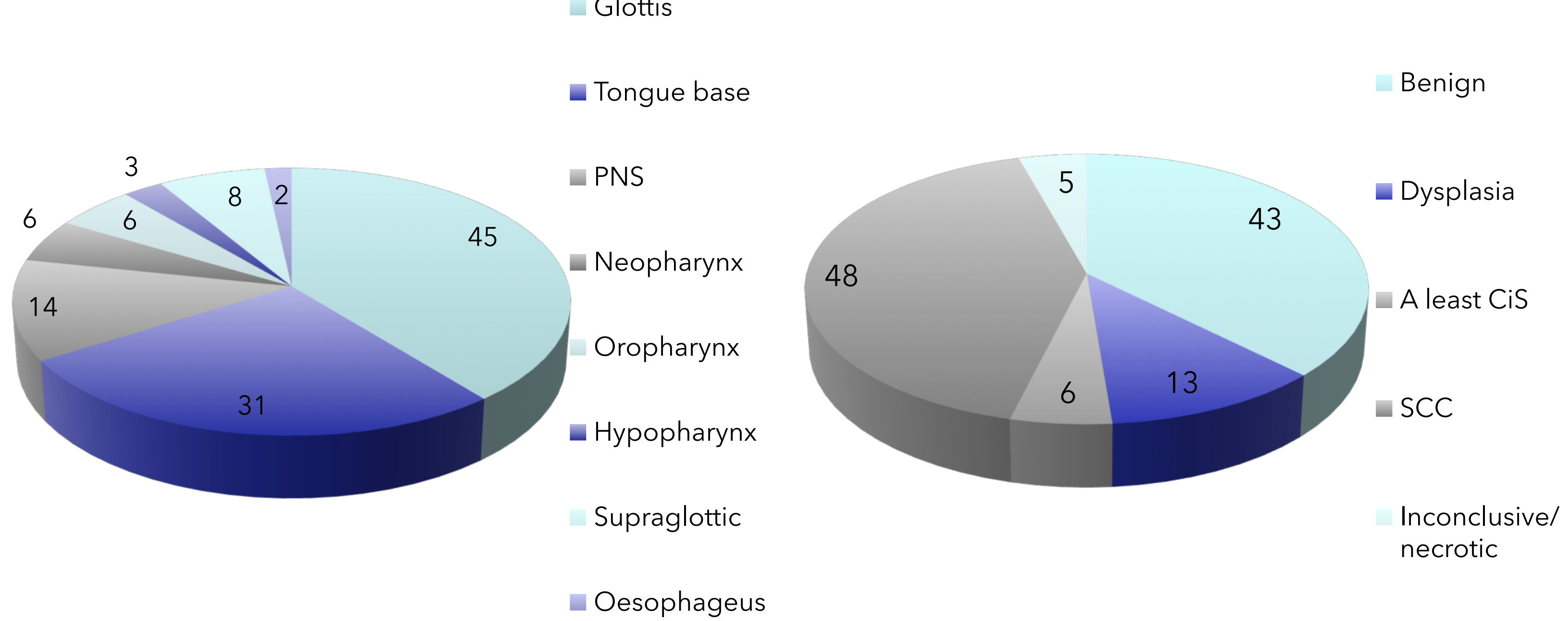

TNO-guided biopsy findings

\section{Conclusion:}

TNO-guided biopsy might be useful as a diagnostic tool for patients suspected of having carcinoma of UADT. Its yield is comparable to traditional diagnostic procedures that require general anaesthesia with better safety profile. 